\title{
EDITORIAL POINT OF VIEW
}

\section{Quantification of Coronary Flow Reserve by ${ }^{15} \mathrm{O}$-Water PET with ATP Stress; from a Practical Application Perspective}

Masao Miyagawa, MD, PhD, Emiri Miyauchi, MD, Hayato Ishimura, RT, Yuki Tanabe, MD, Teruhito Kido, MD, PhD, Akira Kurata, MD, PhD and Teruhito Mochizuki, MD, PhD.

Received: July 11, 2016/Revised manuscript received: July 19, 2016/Accepted: July 19, 2016

(C) The Japanese Society of Nuclear Cardiology 2016

\section{Abstract}

${ }^{15} \mathrm{O}$-water has been considered to be a near-perfect and the most ideal myocardial blood flow (MBF) tracer because it is freely diffusible, metabolically inert, and independent of the myocardial metabolic state, which results in the highest extraction fraction. Absolute coronary flow reserve (CFR) is the ratio of MBF during maximal hyperemia in a coronary artery to $\mathrm{MBF}$ in the same artery under resting conditions and can be quantified noninvasively by positron emission tomography (PET). A growing body of literature is accumulating to show that the prognostic value of absolute MBF or CFR, which is quantified by cardiac PET.

ATP infusion protocol of $0.16 \mathrm{mg} / \mathrm{kg} / \mathrm{min}$ for 5 minutes and its safety profile have been established in humans and it has been widely applied in many clinical and investigative studies including ${ }^{15} \mathrm{O}$-water PET. With the use of the 3 -min acquisition data, the regions of interest in the left ventricular chamber and myocardium could be set for all of the subjects. Six-min CFR data could be used to separate the CAD patients and controls. A 3-min, but not 2min, scan with ${ }^{15} \mathrm{O}$-water PET can be used for the quantitative evaluation of MBF and CFR. A shorter scan time will result in a reduction of body motion of patients, which may lead to the more precise quantification of MBF and CFR.

Keywords: ${ }^{15} \mathrm{O}-\mathrm{H}_{2} \mathrm{O}$, ATP, Coronary flow reserve, PET

Ann Nucl Cardiol $2016 ; 2$ (1) : 58-60

\section{See page 30}

Q uantification of myocardial blood flow (MBF) from dynamic positron emission tomography (PET) is a wellestablished method. ${ }^{15} \mathrm{O}$-labeled $\mathrm{H}_{2} \mathrm{O} \quad\left({ }^{15} \mathrm{O}\right.$-water $),{ }^{13} \mathrm{~N}$ ammonia and ${ }^{82}$ rubidium are the PET tracers commonly used in the assessment of MBF. Among them, ${ }^{15} \mathrm{O}$-water is a nearperfect and the most ideal MBF tracer because it is freely diffusible, metabolically inert, and independent of the myocardial metabolic state, which results in the highest extraction fraction. It has a short half-life of approximately 2 minutes and requires an expensive onsite cyclotron. A practical disadvantage of ${ }^{15} \mathrm{O}$-water is its relatively poor image quality, since it fails to accumulate enough in the myocardium. Additionally, it is not covered by insurance, therefore mainly used for research purposes. Japan Radioisotope Association recommended the continuous infusion of $120-480 \mathrm{MBq} / \mathrm{min}$ (or 2-8 MBq/ $\mathrm{kg} / \mathrm{min}$ ) for ${ }^{15} \mathrm{O}$-water with radiation exposure of $0.9 \mu \mathrm{Sv} / \mathrm{MBq}$ (1), although there is no specific details of ${ }^{15} \mathrm{O}-$ water usage in the latest PET guidelines by the American Society of Nuclear Cardiology (2).

Absolute coronary flow reserve (CFR) is the ratio of MBF during maximal hyperemia in a coronary artery to MBF in the same artery under resting conditions (3). Absolute CFR can be quantified invasively using intracoronary Doppler-based technique or thermo-dilution flow measurements, as well as noninvasively by PET. A growing body of literature is accumulating to show the prognostic value of absolute MBF or CFR, which is quantified by cardiac PET. Briefly, an intact CFR, which has been reported as $>2.5$ with ${ }^{15} \mathrm{O}$-water, is

\section{doi : 10.17996/ANC.02.01.58}

Masao Miyagawa, Emiri Miyauchi, Hayato Ishimura, Yuki Tanabe, Teruhito Kido, Akira Kurata, Teruhito Mochizuki

Department of Radiology, Ehime University Graduate School of

Medicine, Shitsukawa, Toon, Ehime, Japan 791-0295

E-mail: miyagawa@m.ehime-u.ac.jp 
associated with a favorable prognosis. On the other hand, a reduced CFR leads to a worse prognosis either through a severe, focal abnormality with future risk of an acute coronary syndrome, or through a global flow reduction which is a marker for diffuse coronary artery disease (CAD) and its overall burden.

Maruo et al. demonstrate that estimated CFR value of 6min acquisition data by ${ }^{15} \mathrm{O}$-water PET was $4.16 \pm 1.39$ in healthy subjects, while it was $2.19 \pm 0.92$ in patients suspected of having CAD (4). These values are not significantly different to the CFR values of 3-min data and nearly coincide with those that have been reported so far.

\section{Pharmacological stress with ATP for dynamic ${ }^{15} \mathrm{O}$-water PET}

For the pharmacological stress, they infused ATP into an antecubital vein and the PET scanning started at 3 min after the beginning of infusion. Once ATP is injected into the peripheral vein, it is rapidly hydrolyzed to adenosine diphosphate, followed by dephosphorylated AMP with a halflife of $<20$ seconds in plasma. In the animal study, most ATP $(>95 \%)$ is finally degraded to adenosine on the way from a peripheral vein to the coronary sinus. Its degradation product, adenosine, can induce maximal coronary vasodilation through activation of the $\mathrm{A} 2 \mathrm{a}$ receptors with a rapid onset of action and a very short plasma half-life ( $<2$ seconds). Since ATP is a precursor of adenosine, it would be expected to have longer vasodilatory effects than adenosine. ATP infusion protocol of $0.16 \mathrm{mg} / \mathrm{kg} / \mathrm{min}$ for 5 minutes and its safety profile have been established in humans (5-8). Although it is not covered by insurance, ATP stress is feasible and inexpensive $(<10$ dollars per study), therefore it has been widely applied in clinical and investigative studies $(9,10)$.

Maruo et al. reported that there were no significant differences between the values of hemodynamics among the 2min, 3-min and 6-min after the start of the scan, which are, in other words, among the 5-min, 6-min and 9-min after the start of ATP infusion, including the heart rate (HR), systolic blood pressure (SBP), diastolic blood pressure (DBP), and rate pressure product (RPP). Those are substantially similar to the results achieved in the previous reports (5-8) and the maximal coronary vasodilation should be accomplished throughout their study period (Fig. 1).

\section{Shorter scan time is feasible for quantitative ${ }^{15} \mathrm{O}$-water PET}

However, for analysis of the 2-min data, they found difficulties to set proper region of interest (ROI) on the left ventricular (LV) myocardium in $26.7 \%$ of the resting images and $13.3 \%$ of the stress images due to the poor quality of images. ${ }^{15} \mathrm{O}$-water is intravenously injected in a slow-infusion
Quantitative ${ }^{15} \mathrm{O}$-Water PET with ATP

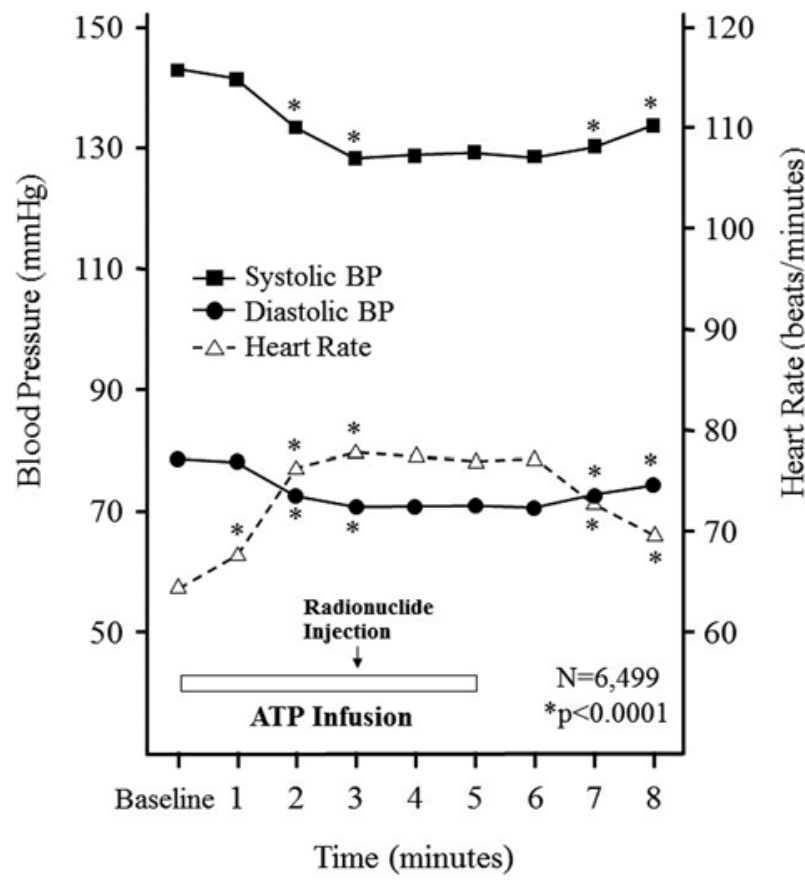

Fig. 1

The mean heart rate and blood pressure response at baseline, during, and after the 5- minute infusion protocol of ATP in 6,499 patients (6). Heart rate increased from the baseline of $64.3 \pm 11.2$ to the maximum of $81.8 \pm 13.4$ beats $/ \mathrm{min}$, while systolic blood pressure (BP) decreased from $142.4 \pm 24.7$ to $117.9 \pm 19.2 \mathrm{mmHg}$ and diastolic BP decreased from $78.5 \pm 14.0$ to $62.6 \pm 11.1 \mathrm{mmHg}$. Hemodynamic effects of ATP infusion reach the plateau at 3 minute after the beginning of infusion and last 3 to 6 minutes.

*: significantly different to the prior time point.

manner for 2-min and there are some delay time to reach the accumulation level of high enough to draw ROIs over the whole LV myocardium. Thus, the low accumulation of whole LV due to the low MBF may be the cause of difficulties in the considerable number of patients.

Meanwhile, the 9-min infusion of ATP seems too long for patients to tolerate. Even with the use of standard 5-min ATP infusion protocol, patients often complain of a chest pain, flushing, headache, neck discomfort, and dyspnea. Moreover, a few patients have high-degree atrioventricular block or ST depression on ECG, which result in early termination of the infusion protocol (8). The body movements of patients can be a source of quantitative errors. Such movement may be problematic when scans are carried out for a relatively long period. Maruo et al. showed that the acquisition time of ${ }^{15} \mathrm{O}$ water PET can be decreased from 6 to 3 min together with that the ATP infusion time can be decreased from 9 to $6 \mathrm{~min}$. A shorter scan time will result in a reduction of body motion of patients, which may lead to the more precise quantification of MBF and CFR. Their innovation in ${ }^{15} \mathrm{O}$-water PET will be of great help for many patients as well as physicians in the field. 


\section{Acknowledgments}

None

\section{Sources of funding}

None

\section{Conflicts of interest}

None declared.

Reprint requests and correspondence:

Masao Miyagawa, MD, PhD

Department of Radiology, Ehime University Graduate School of Medicine, Shitsukawa, Toon, Ehime, Japan 7910295

E-mail: miyagawa@m.ehime-u.ac.jp

\section{References}

1. Japan Radioisotope Association. Guidelines and recommendations for practices of their clinical use of standards of compounds labeled with positron emitting radionuclides approved as established techniques (2009 version). Radioisotopes 2009; 58: 443-54. [in Japanese]

2. Dilsizian V, Bacharach SL, Beanlands RS, et al. ASNC imaging guidelines/SNMMI procedure standard for positron emission tomography (PET) nuclear cardiology procedures. J Nucl Cardiol 2016; 23: in press, doi: 10.1007/s12350-0160522-3
3. Gould KL, Johnson NP, Bateman TM, et al. Anatomic versus physiologic assessment of coronary artery disease. J Am Coll Cardiol 2013; 62: 1639-53.

4. Maruo A, Manabe O, Yoshinaga K, et al. Feasibility of quantifying the myocardial blood flow with a shorter acquisition time using ${ }^{15} \mathrm{O}-\mathrm{H}_{2} \mathrm{O}$ PET. Ann Nucl Cardiol 2016; 2 (1): 30-7.

5. Miyagawa M, Kumano S, Sekiya M, et al. Thallium-201 myocardial tomography with intravenous infusion of adenosine triphosphate in diagnosis of coronary artery disease. J Am Coll Cardiol 1995; 26: 1196-201.

6. Watanabe K, Sekiya M, Ikeda S, et al. Comparison of adenosine triphosphate and dipyridamole in diagnosis by thallium-201 myocardial scintigraphy. J Nucl Med 1997; 38 : 577-81.

7. Bruyne BD, Pijls NHJ, Barbato E, et al. Intracoronary and intravenous adenosine 5-triphosphate, adenosine, papaverine, and contrast medium to assess fractional flow reserve in humans. Circulation 2003; 107: 1877-83.

8. Miyagawa M, Higashino H, Nagao M, et al. Safety of ATP stress myocardial perfusion imaging in 6,499 patients with coronary artery disease: a multicenter study. Radiology 2005; 237: Supplement 678P. http://archive.rsna.org/2005/4409833. html

9. Coma-Canella I, Palazuelos J, Bravo N, et al. Myocardial perfusion imaging with adenosine triphosphate predicts the rate of cardiovascular events. J Nucl Cardiol 2006; 13: 31623.

10. Kurata A, Kawaguchi N, Kido T, et al. Qualitative and quantitative assessment of adenosine triphosphate stress whole-heart dynamic myocardial perfusion imaging using 256-slice computed tomography. PLoS One. 2013; 8: e83950. 